\title{
Interpreting BMI with caution in children with Crohn's disease
}

\author{
M. Keshtkaran ${ }^{1,2}$, A. E. Wiskin ${ }^{1,2}$, S. A. Wootton ${ }^{1,2}$ and R. M. Beattie ${ }^{2,3}$ \\ ${ }^{1}$ University of Southampton School of Medicine, ${ }^{2}$ Southampton NIHR Biomedical Research Unit in Nutrition, Diet and \\ Lifestyle and ${ }^{3}$ Paediatric Medical Unit, Southampton University NHS Hospitals Trust, Southampton SO16 6YD
}

Crohn's disease frequently presents in childhood and impacts on linear growth and pubertal development. Growth is routinely monitored alongside disease activity but little attention is given to measures of body composition. BMI is commonly used as a marker of nutritional well-being, but may mask large differences in body composition. This study aimed at exploring the variance in simple markers of fat and lean mass in relation to BMI in a group of children with Crohn's disease.

Height and weight were determined on a single occasion in children attending the Regional Paediatric Gastroenterology Service. Data were converted to Standard Deviation Scores (SDS) using the UK 1990 Growth Charts (LMS growth programme; Harlow Healthcare, South Shields UK; http://www.healthforallchildren.co.uk). In all except for one child, triceps skinfold thickness (TSF) as a marker of fat mass, and mid-arm circumference (MUC) were also measured and used to determine Upper Arm Muscle Area (UMA), as a marker of lean mass. Both TSF and UMA were then expressed as SDS ${ }^{(1)}$. There were no significant gender effects on any variable and so the results are given as both male and female children combined as mean (min-max).

Sixty-three children with Crohn's disease (38 male, 25 female) aged 6.0-17.8 years (mean 13.7 years) in both active disease ( $n$ 37) and in remission ( $n$ 26) were studied. The SDS for height, weight and BMI were -0.54 ( -2.97 to 1.96), -0.57 ( -3.66 to 2.04$)$ and -0.40 ( -4.35 to 2.63 ), respectively. The height of 58 children fell within \pm 2 SDS; 45 of which also had a BMI within \pm 2 SDS, while 8 children ( 3 male, 5 female) had BMI under -2 SDS and 5 children (4 male, 1 female) had BMI over 2 SDS. Five children (all male) had a height under -2 SDS, 3 of which also had a BMI under -2 SDS. The mean TSF SDS was significantly greater than the mean UMA SDS $(0.21(-1.79$ to 3.85$) v$. $-0.86(-3.57$ to 5.30$) ; P<0.001$ paired $t$-test $)$. There were marked differences in TSF SDS and UMA SDS for any given BMI SDS. For a BMI SDS between -0.5 and +0.5, TSF SDS ranged from -1.0 to 3.4 and UMA SDS ranged from -3.3 to 5.3. There were no significant correlations (Spearman) between BMI SDS and either TSF SDS $\left(r^{2} 0.06\right)$ or UMA SDS ( $\left.r^{2} 0.04\right)$.

In this cohort, the majority of children exhibited height and BMI that would not mark nutritional risk. More children would be identified as at increased risk due to a low BMI than low height (13v. 5 children) and only 3 children had both low height and BMI that would mark nutritional risk. There may also be a risk of excess weight or fatness within this same cohort as indicated by high BMI SDS and TSF SDS. However, even within those with a clinically acceptable weight for height, there may be children with a lean deficit and fat excess that would not be immediately evident from measures of height and weight but may be detected using simple anthropometric measures. These observations emphasise the need for a more complete consideration of body composition in the clinical assessment of growth in children with Crohn's disease.

1. Frisancho AR (1990) Anthropometric Standards for the Assessment of Growth and Nutritional Status. Ann Arbour, MI: University of Michigan Press. 